\title{
GREEN SYNTHESIS OF SILVER NANOPARTICLE AND ITS ANTIBACTERIAL ACTIVITY
}

\author{
Chanel Tri Handoko ${ }^{1}$, Adri Huda ${ }^{1}$, Muhammad Djoni Bustan ${ }^{2}$, Bambang \\ Yudono", and Fakhili Gulo, ${ }^{1, *}$ \\ ${ }^{1}$ Program Studi Ilmu Lingkungan, Program Pascasarjana, Universitas Sriwijaya, \\ Palembang-30139, Indonesia \\ ${ }^{2}$ Program Studi Teknik Kimia, Fakultas Teknik, Universitas Sriwijaya, \\ Indralaya-30862, Indonesia \\ *E-mail: fgulo@unsri.ac.id
}

\begin{abstract}
Silver nanoparticle (Ag-np) was successfully synthesized by using the simple green chemistry method. Its antibacterial activity was studied against Staphylococcus aureus and Escherichia coli according to agar well diffusion method. The green synthesis was carried out by mixing the silver nitrate, glucose, and starch as precursors at $90^{\circ} \mathrm{C}$ for 4 hours. The UV-Vis spectra show the characteristic of spherical Ag-np in the range of 410-425 nm and it is confirmed by TEM image. The Ag-np has an average diameter of $8.41 \pm 4.37 \mathrm{~nm}$. As the reductant mole composition increases, the SPR decreases and shifts to red due to the further growth of the Ag-np. When the stabilizer composition increases, the SPR intensity also increases and shifts to red. The formed Ag-np is stable even after one-month synthesis. The antibacterial test on the Ag-np was carried out against gram-positive bacteria (Staphylococcus aureus) and gram-negative bacteria (Escherichia coli). The result shows that a higher concentration of Ag-np is required to kill Staphylococcus aureus compared to Escherichia coli.
\end{abstract}

Keywords: antibacterial, glucose, green synthesis, silver nanoparticles, starch

(c) RASĀYAN. All rights reserved

\section{INTRODUCTION}

The nanotechnology has been developed in recent years due to the wide application in the human beings. It can be applied in electronic devices ${ }^{1}$, health cares ${ }^{2}$, industrial chemistry ${ }^{3}$, etc. The used material in nanotechnology is called the nanomaterials. The nanomaterials are the materials with $1-100 \mathrm{~nm}$ in size. One of the famous nanomaterials is silver nanoparticles (Ag-np). The Ag-np has been reported to have strong antibacterial activity. Nowadays there are many environmental problems and illness caused by the microbial pathogen. Thus, the researchers develop many methods to save the environment from the microbial pathogens, include utilization of Ag-np. For a long time ago, the researchers reported that Agnp can kill until 650 pathogen microorganism, include bacteria, viruses, parasites, fungi, etc. ${ }^{4}$

The main aspects which influence the activity of Ag-np are the size and the shape of the Ag-np. ${ }^{5}$ Thus, the main effort for controlling the size and the shape of the Ag-np is very critical. The Ag-np size and shape can be controlled with various of synthesis route. There are various methods to synthesize the Agnp. In general, the synthesis method can be divided into 3 (three) methods, namely the physical ${ }^{6}$, chemical $^{7}$, and biological or green chemistry method. ${ }^{8}$ All of these synthesis methods involve the use of reductants and stabilizers as the precursors. Some examples of the common reductant are sodium borohydride, ${ }^{9-10}$ sodium citrate, ${ }^{11}$ dimethyl sulfoxide (DMSO) ${ }^{12}$ ethylene glycol (EG) ${ }^{13}$ formaldehyde, ${ }^{14}$ and ascorbic acid. ${ }^{15}$ While some example of the common stabilizers is a halide, carboxylate, and polymer compounds. ${ }^{16-18}$

The physical method has several drawbacks, for example, it usually requires high energy consumption and high cost due to the use of some modern equipment. ${ }^{19}$ In the other hand, the chemical method requires inorganic chemical which harmful to the environment. ${ }^{20}$ 
In our study, we developed the simple green chemistry method to synthesize Ag-np. The green chemistry route refers to the method by using the biological template to obtain the Ag-np. The examples of the biological template are bacterial enzymes ${ }^{21}$, fungi enzymes ${ }^{22}$, algae metabolites ${ }^{23}$, plant extracts ${ }^{24}$, etc. We use the biological template due to the environmentally benign method and produce fewer impurities than others. The biotemplate also belongs to renewable resource due to a vast organism which can produce metabolite compound to form the Ag-np. For biological template synthesis, the main functional groups that have a strong effect to reduce the silver ion into silver metal are carboxyl ${ }^{25}$, aldehyde ${ }^{26}$, and hydroxyl, ${ }^{27}$ whereas the active functional groups for stabilizing the Ag-np is usually peptide from proteins. $^{28}$

In this study, we used glucose and starch as the biological template to synthesize the Ag-np. We used those biological templates because it is easily encountered in the daily life. Raveendran, Fu, and Wallen (2003) have used glucose and starch to synthesize the Ag-np. ${ }^{29}$ They used 0,01 mmol of $\mathrm{AgNO}_{3}, 0,015$ mmol of glucose solution, and $6 \mathrm{~mL}$ of $0,17 \%$ wt starch to prepare the Ag-np. The synthesis condition was $40^{\circ} \mathrm{C}$ and 20 hours of stirring time. As a result, the SPR intensity of the formed Ag-np was around $420 \mathrm{~nm}$. The average particle diameter was $5.3 \pm 2.6 \mathrm{~nm}$. Firstly, in our study, we used shorter reaction time by increasing the reaction temperature to synthesize the Ag-np. Then, we also varied each precursors composition to study the influence of the glucose and starch for preparing the Ag-np. And finally, we will study the antibacterial activity of the Ag-np against gram-positive bacteria (Staphylococcus aureus) and gram-negative bacteria (Escherichia coli).

\section{Materials}

\section{EXPERIMENTAL}

All of the chemicals were used without further purification. Silver nitrate $\left(\mathrm{AgNO}_{3}\right.$, Merck) was used as the silver precursor. $\mathrm{D}(+)$-glucose anhydrous $\left(\mathrm{C}_{6} \mathrm{H}_{12} \mathrm{O}_{6}\right.$, Merck) and starch (Merck) were used as the biological template. The distilled water was used as a solvent. For the antibacterial test, the Staphylococcus aureus (ATCC 25923) and Escherichia coli (ATCC 25922) were provided by Balai Laboratorium Kesehatan Yogyakarta. The Mueller-Hinton agar media was used as a medium for bacterial growth.

\section{Synthesis of Silver Nanoparticles (Ag-np)}

The Ag-np was prepared by following the method of reference ${ }^{29}$ with some modifications. The green synthesis of Ag-np using glucose and starch was achieved through the mixing of silver nitrate $0.025 \mathrm{M}$, glucose $0.05 \mathrm{M}$, and starch $1 \%$ followed by heating at $90-95^{\circ} \mathrm{C}$ for 4 hours. The reaction was carried out in closed and dark condition to avoid a photochemical reaction. The choice of reaction condition was based on the reference ${ }^{30}$. The effect of reductant composition was studied by varying the mole composition of silver nitrate solution and glucose solution 1:1, 1:1.5, and 1:2 respectively. While the variation of starch $1 \%$ solution $10,20,25$, and $30 \mathrm{~mL}$ was applied to study the effect of the stabilizer. The change of the solution was observed from the first hour until four hours after the stirring procedure.

\section{Characterization of the Ag-np}

The color change of the solution was the proof of the Ag-np formation. The surface plasmon resonance was measured by UV-vis spectrophotometer (UV 2400 PC Series, Shimadzu) in interval 200-700 nm. The morphology and the particle size of the formed Ag-np were determined by transmission electron microscope (TEM JEOL JEM 1400) operating at $100 \mathrm{kV}$. The TEM samples were prepared by placing a drop of the Ag-np on carbon-coated copper grids followed by solvent evaporation at room temperature.

\section{Antibacterial Test}

The antibacterial activity of the Ag-np was performed by agar well diffusion (AWD). ${ }^{31,32}$ The amount of $0.5 \mathrm{McF}$ fresh colonies of Staphylococcus aureus and Escherichia coli were spread on Mueller-Hinton agar plates. The holes made in agar with $5 \mathrm{~mm}$ diameter were aseptically filled with $50 \mu \mathrm{L}$ of Ag-np in different concentrations $(0.25,0.5,1,6.25,12.5,25,50$, and $100 \mu \mathrm{g} / \mathrm{mL})$. Furthermore, the plates were 
RASĀYAN J. Chem.

Vol. 10 | No. 4 |1137-1144 | October - December | 2017

incubated at $36 \pm 1{ }^{\circ} \mathrm{C}$ for $24 \mathrm{~h}$ and the zone of inhibition was measured (in millimeters) under reflected light.

\section{UV-Visible Absorption Studies}

\section{RESULTS AND DISCUSSION}

A study of UV-Vis spectra was carried out in the range of 200-700 $\mathrm{nm}$ to determine the surface plasmon resonance (SPR) of the synthesized Ag-np. All of the compounds undergo the change in color from clear solution (initial mixture)-yellowish-brown to dark brown (the final product). The color changing from a clear solution to brown indicates the formation of the Ag-np.

All of the synthesized Ag-np have the SPR value in the range of 410-425 nm. This specific wavelength corresponds to the Ag-np plasmon resonance. The past studies revealed that the SPR value for the Ag-np was in the range $410-450 \mathrm{~nm}$ and attributed to the spherical Ag-np. ${ }^{33-35}$ The TEM image from this study confirms the spherical shape of the Ag-np.

The effect of the silver nitrate to reductant composition and also the stabilizer composition to synthesize the Ag-np was investigate in this study.

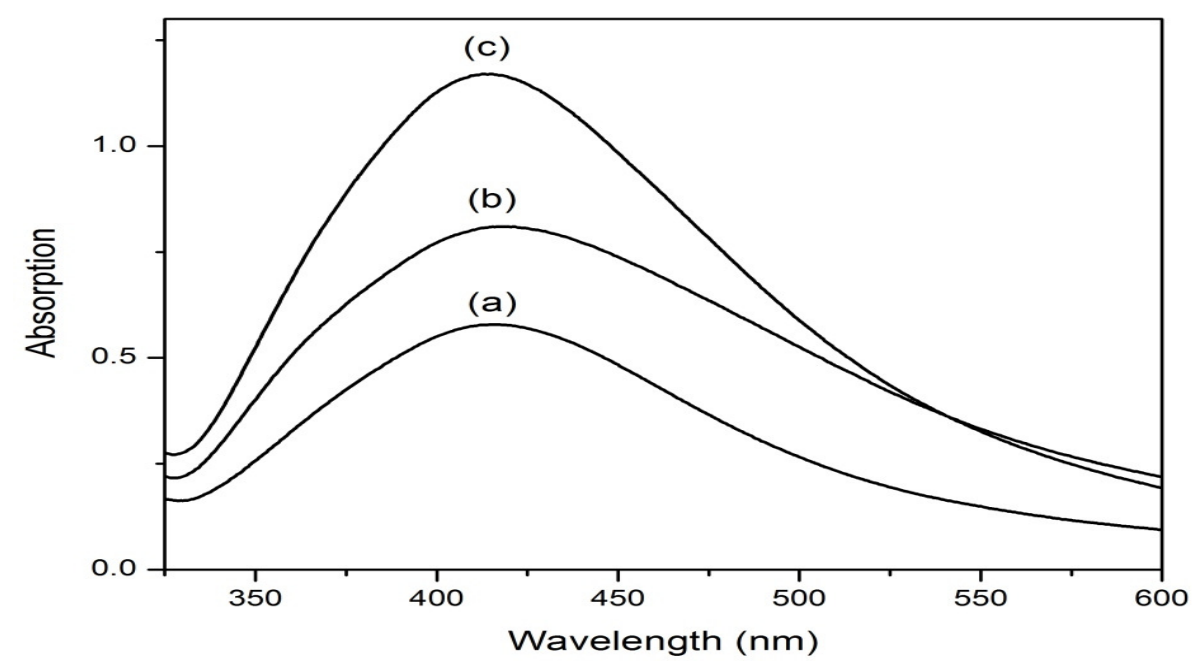

Fig.-1: Effect of the silver nitrate to glucose mole composition for Ag-np synthesis. (a) 1:2, (b) 1:1.5, and (c) 1:1

As shown in Figure-1, the comparison of UV-Vis spectra from different glucose (reductant) composition was studied. In general, it can be seen that increasing the glucose composition gives the effect on decreasing the SPR absorption intensity. The SPR value is $415.50,418.50$, and $419 \mathrm{~nm}$ for the composition 1:1, 1:1.5, and 1:2 of silver nitrate : glucose, respectively. Increasing the glucose concentration contributes to the red-shift effect of the SPR. The literature reveals that the red-shift effect corresponds to the larger Ag-np size. ${ }^{36}$

The influence of glucose composition can be described as follows. The excess of glucose initiates the further growth of the particles. Thus, this phenomenon triggers the increasing SPR value. As the excess of glucose triggers the growth of the particle, the intensity of SPR decreases due to less new particles produced through this reaction. The maximum SPR intensity (1.17) occurred at 1:1 of silver nitrate to glucose composition and the lowest (0.58) occurred at $1: 2$ of silver nitrate to glucose composition.

To study the effect of starch addition, we varied the amount of starch solution $1 \%$ from $10 \mathrm{~mL}$ to $30 \mathrm{~mL}$. As the starch volume increases, the SPR absorption intensity also increases (Figure 2). The intensity of SPR absorption are 1.28, 1.24, 0.89, and 0.63 for $30 \mathrm{~mL}, 25 \mathrm{~mL}, 20 \mathrm{~mL}$, and $10 \mathrm{~mL}$ addition of starch $1 \%$ solution, respectively. This phenomenon is due to the increasing aldehyde functional group during synthesis reaction. The starch as glucose polymer can produce aldehyde through hydrolysis reaction when the starch solution is heated. The aldehyde is a strong reductant for silver ions. Thus, as the starch volume increases, the more new Ag-np is produced. 
From Figure 2 it can be seen that there is an only slight increase in SPR intensity by adding the starch volume from $25 \mathrm{~mL}$ to $30 \mathrm{~mL}$. It indicates that addition of more starch does not have a significant effect on SPR values.

Furthermore, while the amount of the starch increases, the amount of hydroxyl functional groups increases too. The hydroxyl functional group has a role to cap the surface of the Ag-np. The Ag-np surface is stabilized by the hydroxyl group, then the excess of aldehyde has a role to reduce the other silver ion and to produce new particle. For this reason, it offers a theory that the hydroxyl group contributes to increasing the SPR intensity.

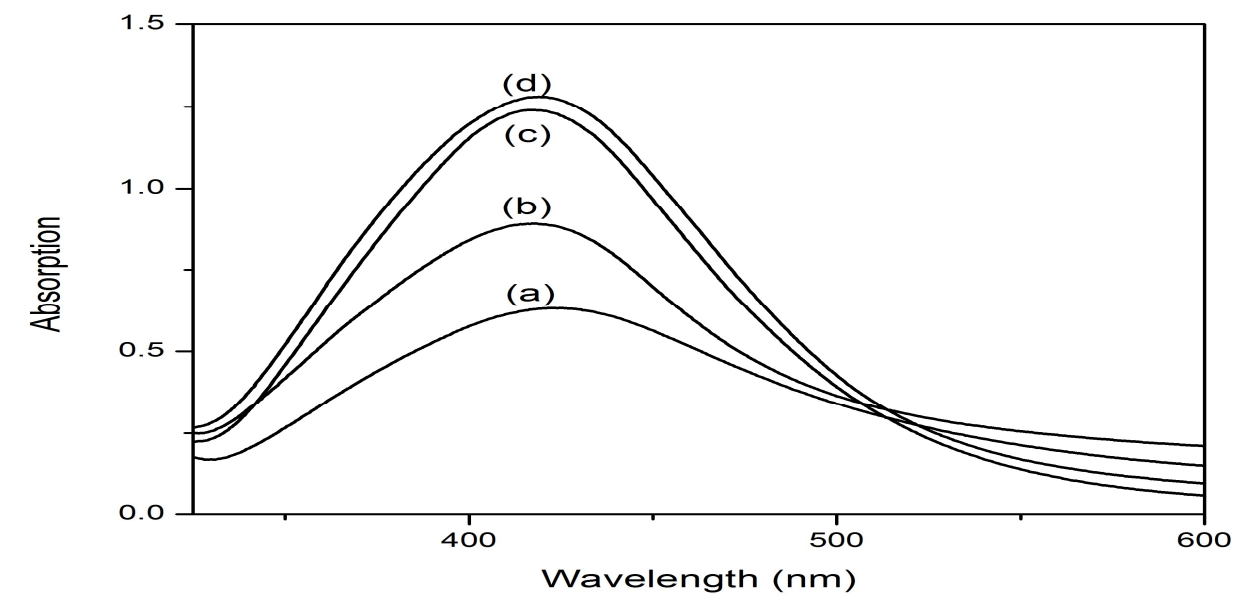

Fig.-2: Effect of the stabilizer volume for Ag-np synthesis(a)10 mL, (b)20 mL, (c)25 mL and (d)30 mL of starch $1 \%$

The SPR shows a sharp peak at 419, 417.50, 417, and $421.50 \mathrm{~nm}$ for $30,25,20$, and $10 \mathrm{~mL}$ of starch solution $1 \%$, respectively. This tendency indicates that increasing the starch volume contributes to the red-shift effect of SPR. The red-shift effect corresponds to the larger Ag-np size. ${ }^{36}$

The explanation of the red-shift phenomenon with the addition of starch solution can be described as follows. In fact, the main function of starch is stabilizer or capping agent. The hydroxyl groups of starch bind with the Ag-np and make a steric repulsion. Thus, it can avoid the agglomeration of the Ag-np. If the agglomeration can be minimized, the smaller Ag-np will be produced. In the other hand, the other fact reveals that when the amount of the starch increases, the amount of aldehyde compound increases too. As described before, the other effect of aldehyde excess can trigger the particles growth of the Ag-np. From the UV-Vis spectra can be inferred that the slight shift phenomenon of SPR due to increasing the aldehyde compound.

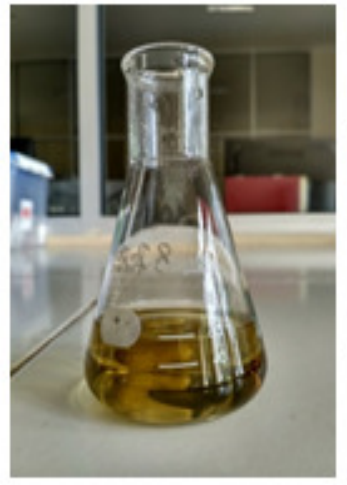

(a)

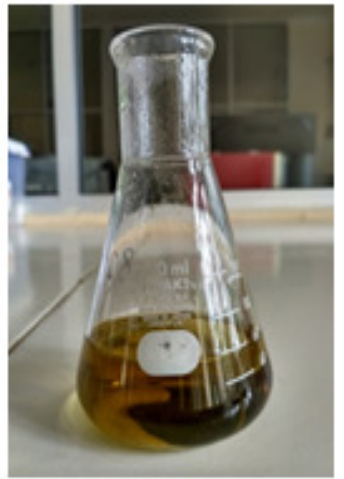

(b)

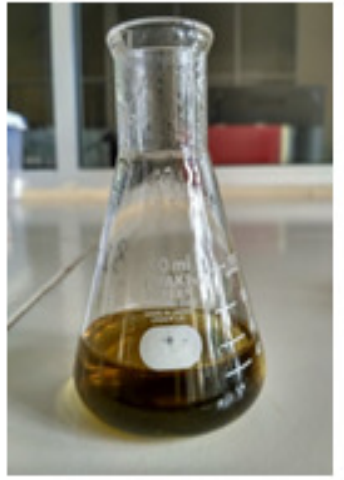

(c)

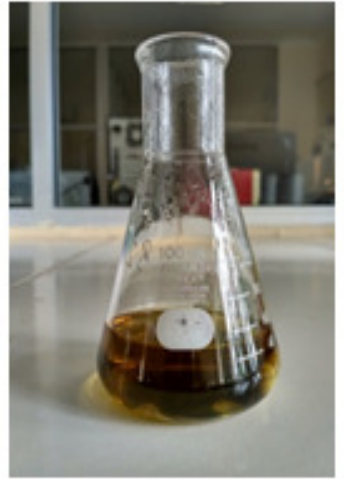

(d)

Fig.-3: The color change of Ag-np after (a) 1 hour, (b) 2 hours, (c) 3 hours), and (d) 4 hours synthesis 
The exception is a sample with the starch amount of $10 \mathrm{~mL}$. Because of the lack amount of the hydroxyl groups of starch, the aldehyde has the major role in the reaction and triggers the growth of the nanoparticle. Thus, with the amount of starch solution $10 \mathrm{~mL}$, the SPR is red-shifted due to the larger nanoparticle are produced.

A sample with silver nitrate to reductant ratio $2: 1$ and starch amount of $20 \mathrm{~mL}$ was used to study the time effect for Ag-np preparation. The time dependent for Ag-np preparation reveals that the SPR value does not change from 1-hour reaction to 4 hours reaction (Fig.-3). The reaction time only influences the SPR absorption and no effect for the SPR shifting. The SPR absorption becomes larger with increasing the reaction time.

\section{TEM Analysis}

The spherical shape of the formed Ag-np was revealed by the Transmission Electron Microscopy (TEM) image. Figure 4 shows the TEM image of Ag-np in various magnifications.

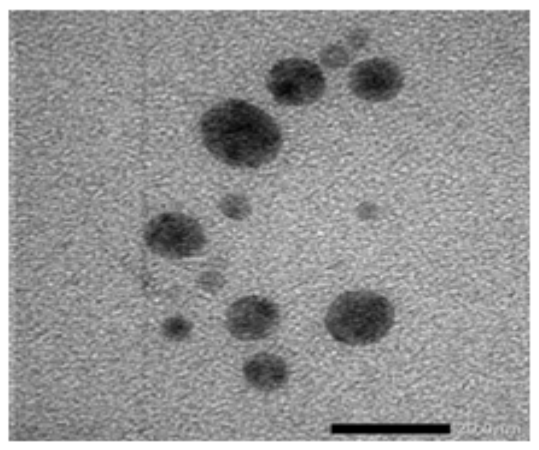

(a)

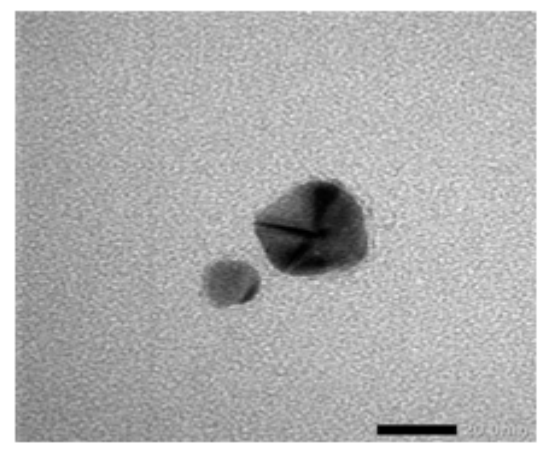

(b)

Fig.-4: The TEM image of the synthesized Ag-np with (a) 300,000 and (b) 200,000 of magnifications

The average size diameter of the formed Ag-np is $8.41 \pm 4.37 \mathrm{~nm}$. This TEM image shows that the particles are still in the range of nanometer scale with spherical shape until one-month after synthesis. It is evidence that the starch can stabilize the nanoparticle surface to avoid the further agglomeration. Thus, the stable nanoparticle can be obtained.

Figure-5 shows the histogram distribution of the Ag-np particle. The minimum size is $3.66 \mathrm{~nm}$ while the maximum is $21.12 \mathrm{~nm}$. From this histogram can be seen that the spherical Ag-np are normally distributed even after one-month synthesis. The advantage of using this method compared with other study is that it does not need the addition of $\mathrm{NaOH}$ as $\mathrm{pH}$ accelerator. ${ }^{30,37}$ Thus this method is environmentally benign due to no more inorganic chemical addition.

\section{The Formation Mechanism of Ag-np Using Glucose and Starch as Precursors}

In this study, we use glucose as reductant and starch as the stabilizer. These two natural precursors are used due to the abundant of these materials. The glucose is known as reducing sugar due to the presence of the aldehyde functional groups in its structure. In the Ag-np preparation, the glucose donates its electrons to the silver ions and is oxidized to become gluconic acid, while the silver ions are reduced into silver metallic. ${ }^{38}$

The main functional group of starch stabilizing the Ag-np is a hydroxyl group. We use starch in this study due to the weak binding interaction between the hydroxyl groups and the particle, so it can easily separate with the increase of the temperature. The other advantages are the starch can integrate with the body and has no toxicity. ${ }^{29}$

The starch is a polymeric form of the glucose. The main constituents of the starch are amylose and amylopectin. When the starch solution is heated, the hot water changes amylose and amylopectin into smaller molecules called glucose. This reaction is called as hydrolysis reaction. ${ }^{39}$ Thus, with the green 
synthesis process using the glucose and starch through hydrolysis reaction of starch can obtain more glucose which gives contribution in the oxidation-reduction reaction. While the starch with hydroxyl functional groups acts as the stabilizer.

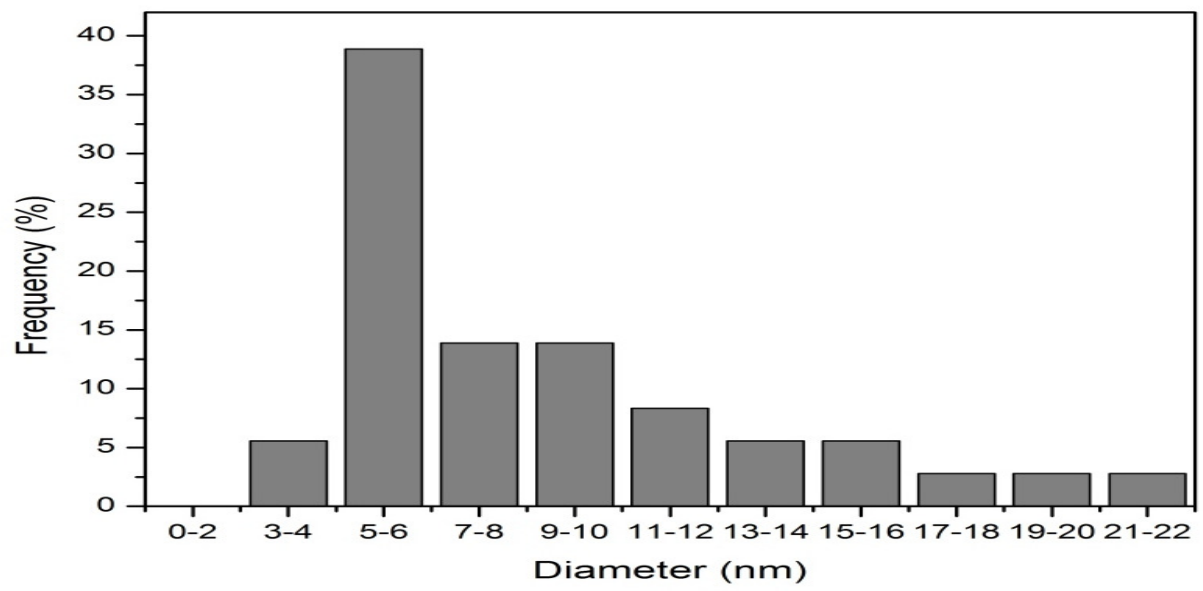

Fig.-5: The histogram of Ag-np particle size

The overall reaction in this prcess is:

$2 \mathrm{AgNO}_{3}(\mathrm{aq})+\mathrm{R}-\mathrm{COH}(\mathrm{aq})+\mathrm{H}_{2} \mathrm{O}(\mathrm{aq}) \rightarrow 2 \mathrm{Ag}(\mathrm{s})+\mathrm{R}-\mathrm{COOH}(\mathrm{aq})+2 \mathrm{HNO}_{3}(\mathrm{aq})$

\section{The Antibacterial Activity Against Staphylococcus aureus and Escherichia coli}

The antibacterial activity of formed Ag-np was investigated using agar well diffusion (AWD) method. The inhibition zone is shown in Table-1. In general, the antibacterial activity of Ag-np increases with increasing the Ag-np concentration. This discovery is in agreement with the previous study on Ag-np antibacterial activity. ${ }^{40}$

Table-1: Inhibition zone of Ag-np against Staphylococcus aureus and Escherichia coli in different concentration

\begin{tabular}{|c|c|c|c|}
\hline \multirow{2}{*}{ No } & \multirow{2}{*}{$\begin{array}{c}\text { Ag-np concentration } \\
(\mu \mathrm{g} / \mathrm{mL})\end{array}$} & \multicolumn{2}{|c|}{ Inhibition zone $(\mathrm{mm})$ against } \\
\hline & & Staphylococcus aureus & Escherichia coli \\
\hline 1. & 0.25 & 0 & 0 \\
\hline 2. & 0.50 & 0 & 0 \\
\hline 3. & 1 & 0 & 7.17 \\
\hline 4. & 6.25 & 0 & 10 \\
\hline 5. & 12.50 & 12.17 & 12.33 \\
\hline 6. & 25 & 15.17 & 14 \\
\hline 7. & 50 & 15.83 & 14.67 \\
\hline 8. & 100 & 17.67 & 15 \\
\hline
\end{tabular}

In this study, variations of Ag-np concentration are $0.25,0.50,1,6.25,12.50,25,50$, and $100 \mu \mathrm{g} / \mathrm{mL}$. No inhibition zone is found at concentration $0.25-6.25 \mu \mathrm{g} / \mathrm{mL}$ against Staphylococcus aureus and at concentration $0.25-0.50 \mu \mathrm{g} / \mathrm{mL}$ against Escherichia coli. When the Ag-np concentration is increased from 12.50 to $100 \mu \mathrm{g} / \mathrm{mL}$, the inhibition zone against Staphylococcus aureus gradually increases. The same observation also occurs against Escherichia coli. When the Ag-np concentration is increased from 1 to $100 \mu \mathrm{g} / \mathrm{mL}$, the inhibition zone will be seen more clearly. The minimum concentration of Ag-np in this study is $12.5 \mu \mathrm{g} / \mathrm{mL}$ to inhibit Staphylococcus aureus and $1 \mu \mathrm{g} / \mathrm{mL}$ to inhibit Escherichia coli.

This result is also in agreement with previous study. ${ }^{41}$ As gram-positive bacteria, Staphylococcus aureus is less susceptible than Escherichia coli (gram-negative) bacteria on Ag-np. This is due to the thicker cell wall and more peptidoglycan in the structure of gram-positive bacteria than that of gram-negative 
bacteria. Thus, a higher concentration of Ag-np is required to kill Staphylococcus aureus compared to Escherichia coli.

The Ag-np activity against the microbial pathogens (for example Staphylococcus aureus and Escherichia coli) has a proposed mechanism. In this mechanism, the silver ions are released during the interaction of Ag-np with water or tissue fluid. The silver ions will penetrate the cell membrane and bound with the electron donor functional groups such as phosphates, thiols, and indoles which contain sulfur and phosphorous compounds. All of these compounds are a presence in DNA or ribosomes. Thus the DNA function will be disrupted and the DNA cannot replicate. As a result, the microbial cell will not grow and eventually die. ${ }^{42}$

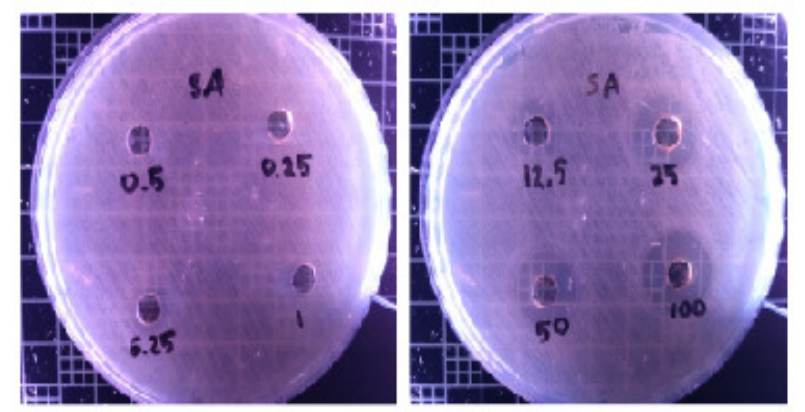

(a)
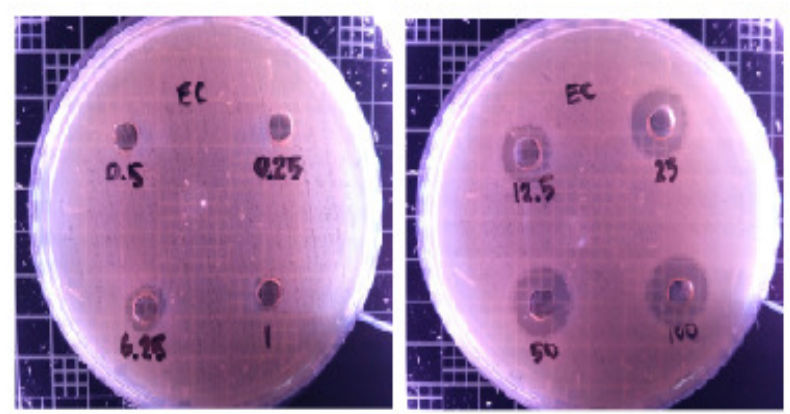

(b)

Fig.-6: Antibacterial activity of Ag-np in different concentration against (a) Staphylococcus aureus and (b) Escherichia coli

\section{CONCLUSION}

The Ag-np with the average diameter size of $8.41 \pm 4.37 \mathrm{~nm}$ was successfully synthesized by using simple green chemistry method with silver nitrate, glucose, and starch as precursors. The UV-Vis spectra show the characteristic of spherical Ag-np in the range of 410-425 nm. From this study, as the reductant mole composition increases, the SPR decreases and shifts to red due to the further growth of the Ag-np. When the stabilizer composition increases, the SPR intensity also increases and shifts to red. The inhibitory effect of formed Ag-np on Escherichia coli is stronger than that on Staphylococcus aureus. A higher concentration of Ag-np is required to killing Staphylococcus aureus compared to Escherichia coli. These results suggest that Ag-np can be used as effective growth inhibitors to both gram-positive and gram-negative bacteria.

\section{ACKNOWLEDGEMENT}

The authors would like to thank the Ministry of Research, Technology, and Higher Education of Indonesia and Balai Riset dan Standardisasi Industri (Baristand) Palembang, which have provided the facilities for our study.

\section{REFERENCES}

1. G. Polino, R. Abbel, S. Shanmugam, G. J. P. Bex, R. Hendriks, F. Brunetti, A. Di Carlo, R. Andriessen and Y. Galagan, Org. Electron., 34, 130 (2016).

2. M. Yao, L. Ma, L. Li, J. Zhang, R. X. Lim, W. Chen and Y. Zhang, J. Biomed. Nanotechnol., 12(10), 1835 (2016).

3. A. Haider and I. K. Kang, Adv. Mater. Sci. Eng., 2015, 1 (2015).

4. S. J. Kang, Y. Il Cho, K. H. Kim and E. S. Cho, Biol. Trace Elem. Res., 171(1), 101 (2016).

5. M. Raza, Z. Kanwal, A. Rauf, A. Sabri, S. Riaz and S. Naseem, Nanomaterials, 6(4), 74 (2016).

6. G. R. Khayati and K. Janghorban, Adv. Powder Technol., 23(3), 393 (2012).

7. G. Suriati, M. Mariatti and A. Azizan, International Journal of Automotive and Mechanical Engineering, 10(12), 1920 (2014).

8. S. Ahmed, Saifullah, M. Ahmad, B. L. Swami and S. Ikram, J. Radiat. Res. Appl. Sci., 9(1), 1 (2016). 
9. M. B. Ahmad, J. J. Lim, K. Shameli, N. A. Ibrahim and M. Y. Tay, Molecules, 16(12), 7237 (2011).

10. K. Sivaram, M. C. Rao, G. Giridhar, M. Tejaswi, B. T. P. Madhav, V. G. K. M. Pisipati and R. K. N. R. Manepalli, Rasayan J. Chem., 9(4), 697 (2017).

11. A. Van Hoonacker and P. Englebienne, Curr. Nanosci., 2(4), 359 (2006).

12. G. Rodríguez-Gattorno, D. Díaz, L. Rendón and G. O. Hernández-Segura, J. Phys. Chem. B, 106(10), 2482 (2002).

13. J. Li, J. Zhu and X. Liu, Dalt. Trans., 43(1), 132 (2014).

14. A. Yang, Z. Zhang, Y. Yang, X. Bao, and R. Yang, Optoelectron. Lett., 9(1), 1 (2013).

15. D. Singha, N. Barman and K. Sahu, J. Colloid Interface Sci., 413, 37 (2014).

16. H. Liu and N. Toshima, J. Chem. Soc. Chem. Commun., 438(16), 1095 (1992).

17. A. C. Templeton, W. P. Wuelfing and R. W. Murray, Acc. Chem. Res., 33(1), 27 (2000).

18. K. Shakti Singh, G. K. Lohiya and V. K. Mourya, Rasayan J. Chem., 2, 451 (2009).

19. J. Natsuki, Int. J. Mater. Sci. Appl., 4(5), 325 (2015).

20. M. Murphy, K. Ting, X. Zhang, C. Soo and Z. Zheng, J. Nanomater., 2015, 1 (2015).

21. S. Sunkar and C. V. Nachiyar, Asian Pac. J. Trop. Biomed., 2(12), 953 (2012).

22. F. Fatima, P. Bajpai, N. Pathak, S. Singh, S. Priya and S. Verma, BMC Microbiol., 15(1), 52 (2015).

23. T. Kathiraven, A. Sundaramanickam, N. Shanmugam and T. Balasubramanian, Appl. Nanosci., 5(4), 499 (2015).

24. G. M. Srirangam and K. Parameswara Rao, Rasayan J. Chem., 10(1), 46 (2017).

25. S. S. Shankar, A. Ahmad and M. Sastry, Biotechnol. Prog., 19(6), 1627 (2003)

26. M. Darroudi, M. Mansor Bin Ahmad, A. H. Abdullah, N. A. Ibrahim and K. Shameli, Int. J. Nanomedicine, 6(1), 569 (2011).

27. N. Ahmad, S. Sharma, M. K. Alam, V. N. Singh, S. F., Shamsi, B. R., Mehta and A. Fatma, Colloids Surfaces B Biointerfaces, 81(1), 81 (2010).

28. R. R. R. Kannan, R. Arumugam, D. Ramya, K. Manivannan and P. Anantharaman, Appl. Nanosci., 3(3), 229 (2013).

29. P. Raveendran, J. Fu and S. L. Wallen, J. Am. Chem. Soc., 125(46), 13940 (2003).

30. L. Ortega-Arroyo, E. S. Martin-Martinez, M. A. Aguilar-Mendez, A. Cruz-Orea, I. Hernandez-Pérez and C. Glorieux, Starch - Stärke, 65, 814 (2013).

31. K. Kalishwaralal, S. BarathManiKanth, S. R. K. Pandian, V. Deepak and S. Gurunathan, Colloids Surfaces B Biointerfaces, 79, 340 (2010).

32. E. D.Cassavin, L. F. P. de Figueiredo, J. P. Otoch, M. M. Secler, R. A. de Oliveira, F. F. Franco, V. S. Marangoni, V. Zucolotto, A. S. S. Levin and S. F. Costa, J. Nanobiotechnology, 13, 64 (2015).

33. S. Maiti, G. Barman and J. K. Laha, Adv. Sci. Focus, 1(2), 145 (2013).

34. S. Maiti, D. Krishnan, G. Barman, S. K. Ghosh and J. K. Laha, J. Anal. Sci. Technol., 5(1), 40 (2014).

35. Z. Zaheer and Rafiuddin, Colloids Surfaces B Biointerfaces, 90, 48 (2012).

36. C. Lee and P. Zhang, J. Raman Spectrosc., 44(6), 823 (2013).

37. G. Ayala Valencia, L. Cristina de Oliveira Vercik, R. Ferrari, and A. Vercik, Starch - Stärke, 65, 931 (2013).

38. M. Darroudi, M. Bin Ahmad, A. H. Abdullah, N. A. Ibrahim, and K. Shameli, Int. J. Mol. Sci., 11(10), 3898 (2010).

39. M. Venkatesham, D. Ayodhya, A. Madhusudhan, A. Santoshi Kumari, G. Veerabhadram, and K. Girija Mangatayaru, J. Clust. Sci., 25(2), 409 (2014).

40. K. Jyoti, M. Baunthiyal and A. Singh, J. Radiat. Res. Appl. Sci., 9, 217 (2016).

41. S. Shrivastava, T. Bera, A. Roy, G. Singh, P. Ramachandrarao and D. Dash, Nanotechnology, 18(22), 225103 (2007).

42. G. Franci, A. Falanga, S. Galdiero, L. Palomba, M. Rai, G. Morelli and M. Galdiero, Molecules, 20, 8856 (2015).

[RJC-1875/2017] 